\title{
Possible multiple evolution of indirect telencephalo-cerebellar pathways in teleosts: studies in Carassius auratus and Pantodon buchholzi
}

\author{
Mario F. Wullimann ${ }^{1}$, Dietrich L. Meyer ${ }^{2}$ \\ I Institut für Hirnforschung, Universität Bremen, FB 2-NW II, Postfach 33 04 40, D-28334 Bremen, Germany \\ ${ }^{2}$ Abteilung Neuroanatomie, Zentrum Anatomie, Universität Göttingen, D-37075 Göttingen, Germany \\ Received: 1 March 1993 / Accepted: 30 April 1993
}

\begin{abstract}
Among vertebrates, telencephalo-pontine systems exist only in birds and mammals. However, three nuclei in the diencephalon and mesencephalon of teleost fishes have been indicated - analogous to the pons - to represent relay stations between telencephalon and cerebellum. Since two of these nuclei (dorsal preglomerular nucleus, dorsal tegmental nucleus) have only been described in the highly derived, electrosensory mormyrids, we investigated telencephalic connections in two nonelectrosensory teleosts, the goldfish Carassius auratus and the freshwater butterflyfish Pantodon buchholzi, and cerebellar connections only in the latter species, since for $C$. auratus these connections are already established. Horseradish peroxidase tracing reveals that $C$. auratus has a dorsal tegmental nucleus and a paracommissural nucleus both of which are telencephalo-recipient and project to the cerebellum, and that $P$. buchholzi has a dorsal preglomerular nucleus with such connections. These results extend our knowlegde of the distribution and, therefore, the phylogeny of telencephalo-cerebellar systems in teleosts. Similar to tetrapods, teleosts appear to have developed telencephalo-cerebellar systems several times independently.
\end{abstract}

Key words: Cerebellum - Evolution - Brain, vertebrate Pons - Telencephalon - Carassius auratus, Pantodon buchholzi (Teleostei)

\section{Introduction}

The telencephalon of mammals and birds exerts influence on the cerebellum via metencephalic pontine nuclei. Comparable systems have not been described in other tetrapods. The distant phylogenetic relationship of these two major vertebrate groups suggests that avian and mammalian telencephalo-pontine systems evolved inde-

Correspondence to: M.F. Wullimann pendently (Roth and Wullimann, in press). In contrast to birds and mammals, teleost fishes lack a pons. However, three nuclei in the diencephalon and mesencephalon of various teleost species (see Discussion) have been indicated as relay stations for telencephalo-cerebellar pathways.

(1) Some euteleosts, the evolutionarily most derived teleost clade (Fig. 5), possess a telencephalo-cerebellar pathway via the nucleus paracommissuralis, which is located most dorsally in the diencephalon (Karten and Finger 1976; Ito et al. 1982; Striedter 1990). The nucleus and the related pathway have only been described in euteleosts (Wullimann and Northcutt 1988). Among euteleosts, however, the cyprinids have been argued to lack a nucleus paracommissuralis (Ito et al. 1982). In contrast, Wullimann and Northcutt (1988) described a minute nucleus paracommissuralis in the cyprinid Carassius auratus, based on its projection to the corpus cerebelli.

(2) A second telencephalo-cerebellar pathway via a dorsal tegmental nucleus in the African electric fish Gnathonemus petersi has been discovered more recently (Meek et al. 1986; Wullimann and Northcutt 1990). There is evidence (see Discussion) that this nucleus receives telencephalic input and projects to the cerebellum in various other teleost species belonging to the osteoglossomorph as well as the euteleost clades. However, it has not been demonstrated that the two connections exist together in a single teleost species except for the highly specialized $G$. petersi.

(3) In addition, G. petersi displays a third telencephalo-cerebellar pathway. A dorsal subdivision of the diencephalic preglomerular area, the dorsal preglomerular nucleus, has a massive terminal field from the telencephalon (Wullimann and Northcutt 1987, 1990). It has previously been shown that this nucleus (dorsal anterior pretectal nucleus of Meek et al. 1986) projects heavily to the corpus cerebelli. This diencephalic telencephalo-cerebellar relay center can reasonably be assumed to be involved in the processing of electrosensory information (i.e., electrolocation), since it receives ascending input from the lateral nucleus of the torus semicircularis, the mesencephalic target of the electrolocative component of 
this sensory modality (Finger et al. 1981). As electroreception evolved newly in mormyrids (Bullock et al. 1983), Wullimann and Northcutt (1990) have interpreted this telencephalo-preglomerulo-cerebellar pathway as a uniquely derived (autapomorphic) feature of mormyrids. However, non-electrosensory osteoglossomorphs have not been investigated for the presence of this pathway.

The goal of the present study is to test further the evolutionary polarity of the three telencephalo-cerebellar systems observed in various teleost species. Since the cerebellar connections have been extensively documented in C. auratus (Wullimann and Northcutt 1988), we chose this ostariophysine species to investigate whether the nucleus paracommissuralis and the dorsal tegmental nucleus are telencephalo-recipient. Further, we investigated the non-electrosensory osteoglossomorph P. buchholzi for the presence of a telencephalo-cerebellar pathway via the dorsal preglomerular nucleus.

The neuroanatomical terminology is that of Braford and Northcutt (1983), as modified by Wullimann and Northcutt (1990) and Wullimann and Meyer (1990).

\section{Materials and methods}

\section{Horseradish peroxidase (HRP) tracing experiments}

Two teleostean species, the goldfish (Carassius auratus) and the freshwater butterflyfish (Pantodon buchholzi) were used in the present study. HRP injections were made into the telencephalon $(C$. auratus: 9 cases; P. buchholzi: 4 cases) or into the cerebellum ( $P$. buchholzi: 2 cases). The animals were deeply anesthetized in a dilute solution of tricaine methanesulfonate (MS 222, Sigma, Deisenhofen, Germany). A small cranial hole was made at the appropriate location and solid HRP (Boehringer, Mannheim, Germany) was pin-injected into the telencephalon or the corpus cerebelli. A piece of Gelfoam (Upjohn) was placed onto the injection site and the wound closed with Histoacryl (Braun, Melsungen, Germany).

After survival times ranging from 1 to 6 days, the animals were re-anesthetized with MS 222 and perfused transcardially with cold teleost Ringer's solution, followed by $4 \%$ glutaraldehyde in $0.1 \mathrm{M}$ phosphate buffer ( $\mathrm{pH}$ 7.4). The brains were removed from the crania, postfixed for $1 \mathrm{~h}$ in the same fixative containing 30\% sucrose and cryprotected overnight in $30 \%$ sucrose-phosphate buffer before they were cut transversely on a cryostat at a thickness of $25 \mu \mathrm{m}$. The sections were reacted according to either the Hanker-Yates protocol (Hanker et al. 1977) or the protocol of Ebbesson et al. (1981). Some sections were counterstained with cresyl violet.

\section{Histological procedures}

One brain from $C$. auratus and $P$. buchholzi was prepared according to the Bodian silver staining method for comparison with the experimental HRP material. Details of the Bodian procedure have been given elsewhere (Wullimann and Northcutt 1988).

\section{Results}

\section{Carassius auratus}

Telencephalic connections. Although the HRP injections into the telencephalon were placed in the central zone of area dorsalis telencephali (telencephalic nomenclature and subdivisions of Nieuwenhuys and Meek 1990), additional portions of the area dorsalis (e.g., the medial and dorsal zones) were also partially affected. After such rather large injections into the dorsal area of the telencephalon, the heavily labeled medial and lateral forebrain bundles can be traced into the diencephalon (Fig. 1a). At this level, the lateral forebrain bundle gives off a large dorsal tract. The latter contains efferent fibers which eventually reach the nucleus paracommissuralis (Fig. 1c), the dorsal tegmental nucleus (Figs. 1d,e;4c) and the central zone of the optic tectum (Fig. 1b-f). Additional efferents, which deviate from the lateral forebrain bundle even more rostrally, reach the ventrolateral thalamic and dorsal posterior thalamic nuclei (Fig. 1a,b). The medial forebrain bundle courses ventrocaudally and reaches the posterior tuberal nucleus, where labeled cells and terminals are observed (Fig. 1d).

The lateral forebrain bundle gives off terminals to the anterior, lateral, and medial preglomerular nuclei. In contrast to $P$. buchholzi, there is no separate telencephalo-recipient preglomerular nucleus dorsal to the lateral preglomerular nucleus. Many retrogradely labeled cells are present in these three preglomerular nuclei in $C$. auratus, and in the posterior thalamic and the subglomerular nuclei (Fig. 1a-e). However, the tertiary gustatory nucleus, the commissural preglomerular nucleus and the corpus mammillare are devoid of label.

Additional efferent projections which run in the lateral forebrain bundle, reach the anterior tuberal nucleus, the nucleus of the lateral torus, the diffuse and, strongly, the central nuclei of the inferior lobe (Fig. 1a-e). Further caudally, the superior raphe and the locus coeruleus are retrogradely labeled (Fig. 1f). All connections are strictly ipsilateral, with the exception of the ones to and from the posterior tuberal nucleus, from the superior raphe and from the locus coeruleus.

\section{Pantodon buchholzi}

Telencephalic connections. The specific connections of most telencephalic subdivisions (e.g., with the preglomerular area) in $P$. buchholzi were recently investigated in detail with the carbocyanine dye Dil (Wullimann and Roth 1992; and unpublished observations) and will be published separately. Here, only HRP-data on telencephalic injections in $P$. buchholzi are reported which selectively relate to nuclei also projecting to the cerebellum. The DiI-data mentioned above confirm these HRP-results.

As in C. auratus, the HRP-injections into the telencephalon of $P$. buchholzi reported here mainly affected the central zone of the area dorsalis telencephali, with the likely inclusion of bordering dorsal telencephalic zones. After such extensive telencephalic HRP-injections, a nucleus that is clearly part of the preglomerular area (Fig. 2), is massively labeled anterogradely throughout its rostrocaudal extent ipsilaterally (Fig. 3c,d). The histological appearance of this somewhat ovally shaped dorsal preglomerular nucleus is characterized by the presence of 

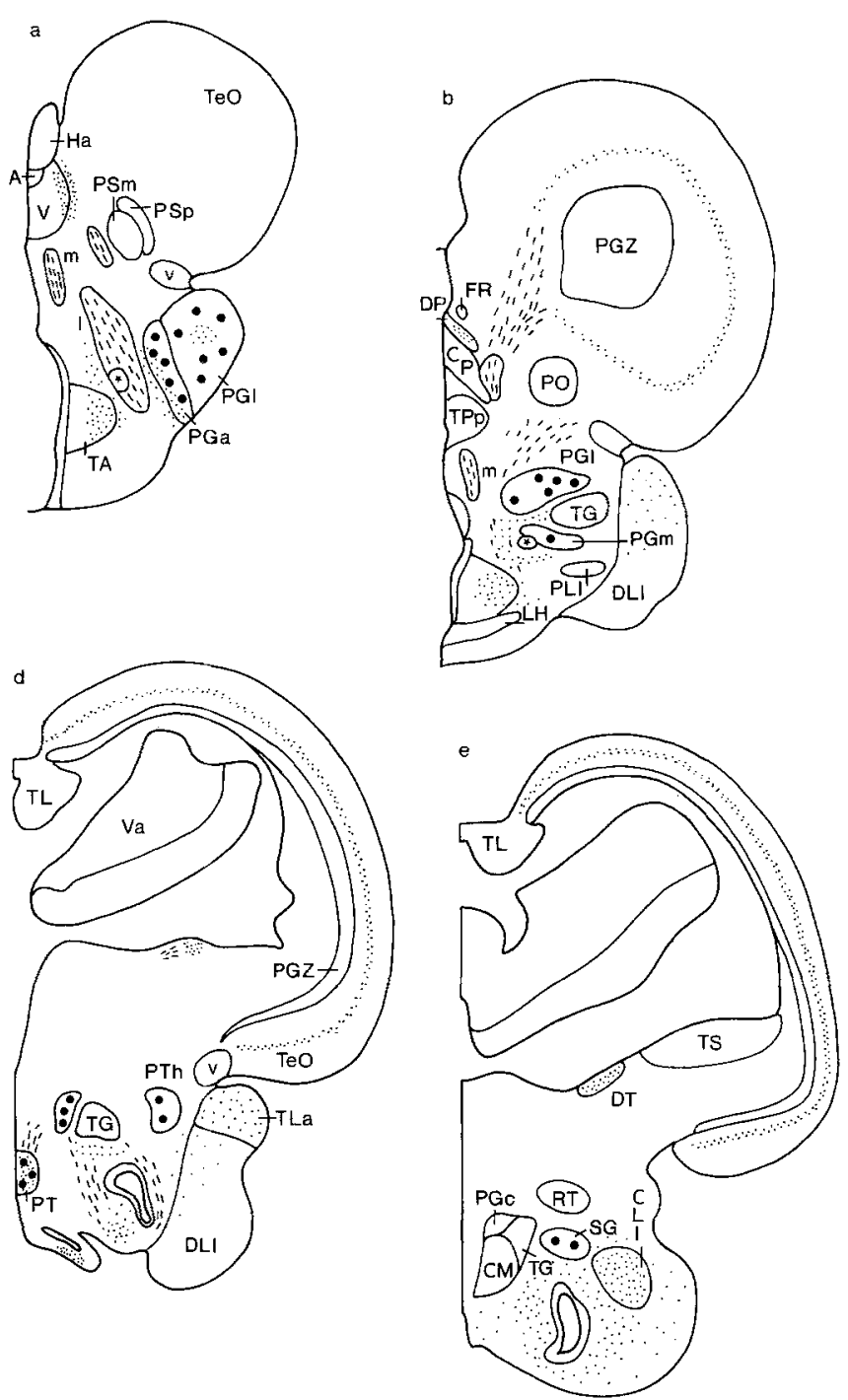

Fig. 1. Telencephalic connections in $C$. auratus are charted into drawings of cross-sections at levels from rostral a to caudal $\mathbf{f} ;$ black dots retrogradely labeled cells; stippling terminal arborizations; dashes labeled fibers. A Anterior thalamic nucleus; $C C$ corpus cerebelli; $C L I$ central nucleus of inferior lobe; $C M$ corpus mammillare; $C P$ central posterior thalamic nucleus; $D L I$ diffuse nucleus of the inferior lobe; $D P$ dorsal posterior thalamic nucleus; $D T$ dorsal tegmental nucleus; $F R$ fasciculus retroflexus; $H a$ habenula; $L C$ locus coeruleus; / lateral forebrain bundle; $L H$ lateral hypothalamic nucleus; $M L F$ medial longitudinal fascicle; $m$ medial forebrain bundle; $P C$ nucleus paracommissuralis; $P C o$ posterior commissure; $P G a$ anterior preglomerular nucleus; $P G c$ commissural preglomerular nucleus; $P G l$ lateral preglomerular nucleus; $P G m$ medial preglomerular nucleus; $P G Z$ periventricular grey zone of optic

many neurons bordering a soma-sparse neuropilar core (Fig. 2).

Unlike $C$. auratus, no telencephalic efferents can be seen to reach a position dorsal to the posterior commissure, where a nucleus paracommissuralis would be expected. Also, no telencephalic terminals are observed in the dorsal tegmental nucleus in the HRP experiments. However, telencephalic terminals can be found in the dorsal tegmental nucleus after injections of DiI into the lateral forebrain bundle of $P$. buchholzi.
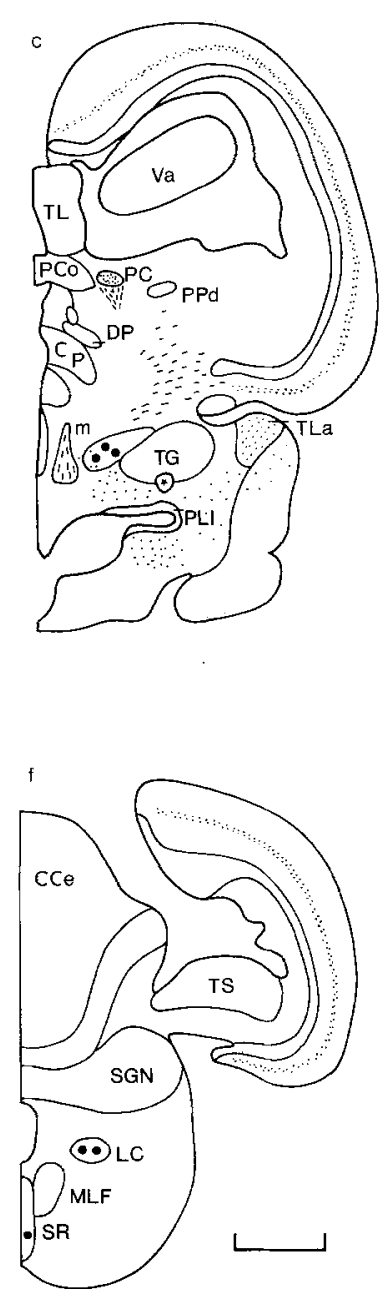

tectum; $P L I$ periventricular nucleus of the lateral recess; $P O$ posterior pretectal nucleus; $P P d$ dorsal part of periventricular pretectal nucleus; $P P v$ ventral part of periventricular pretectal nucleus; $P S m$ magnocellular superficial pretectal nucleus; $P S p$ parvocellular superficial pretectal nucleus; $P T$ posterior tuberal nucleus; $P T h$ posterior thalamic nucleus; $R T$ rostral tegmental nucleus; $S G$ subglomerular nucleus; $S G N$ secondary gustatory nucleus; $S R$ superior raphe; $T A$ anterior tuberal nucleus; $T e O$ optic tectum; $T G$ tertiary gustatory nucleus; $T L$ torus longitudinalis; $T L a$ nucleus of the torus lateralis; $T P p$ periventricular nucleus of the posterior tuberculum; IS torus semicircularis; $V$ ventromedial and ventrolateral thalamic nucleus; $v$ ventrolateral optic tract; $V a$ valvula cerebelli; asterisk horizontal commissure. Lateral is to the right in this and all other figures. Bar: $0.5 \mathrm{~mm}$

Cerebellar connections. After HRP injections into the corpus cerebelli, the observed pattern of nuclei projecting to the corpus cerebelli in $P$. buchholzi is very similar to the one previously reported for two other teleost species (Wullimann and Northcutt 1988). In P. buchholzi, retrogradely labeled neurons were present in pretectal nuclei (dorsal periventricular pretectum, central pretectum), in the accessory optic system (dorsal accessory optic nucleus), in the tegmentum (ventral and dorsal tegmental nuclei, Fig. 4 d,e) and in the brainstem (nucleus lateralis 

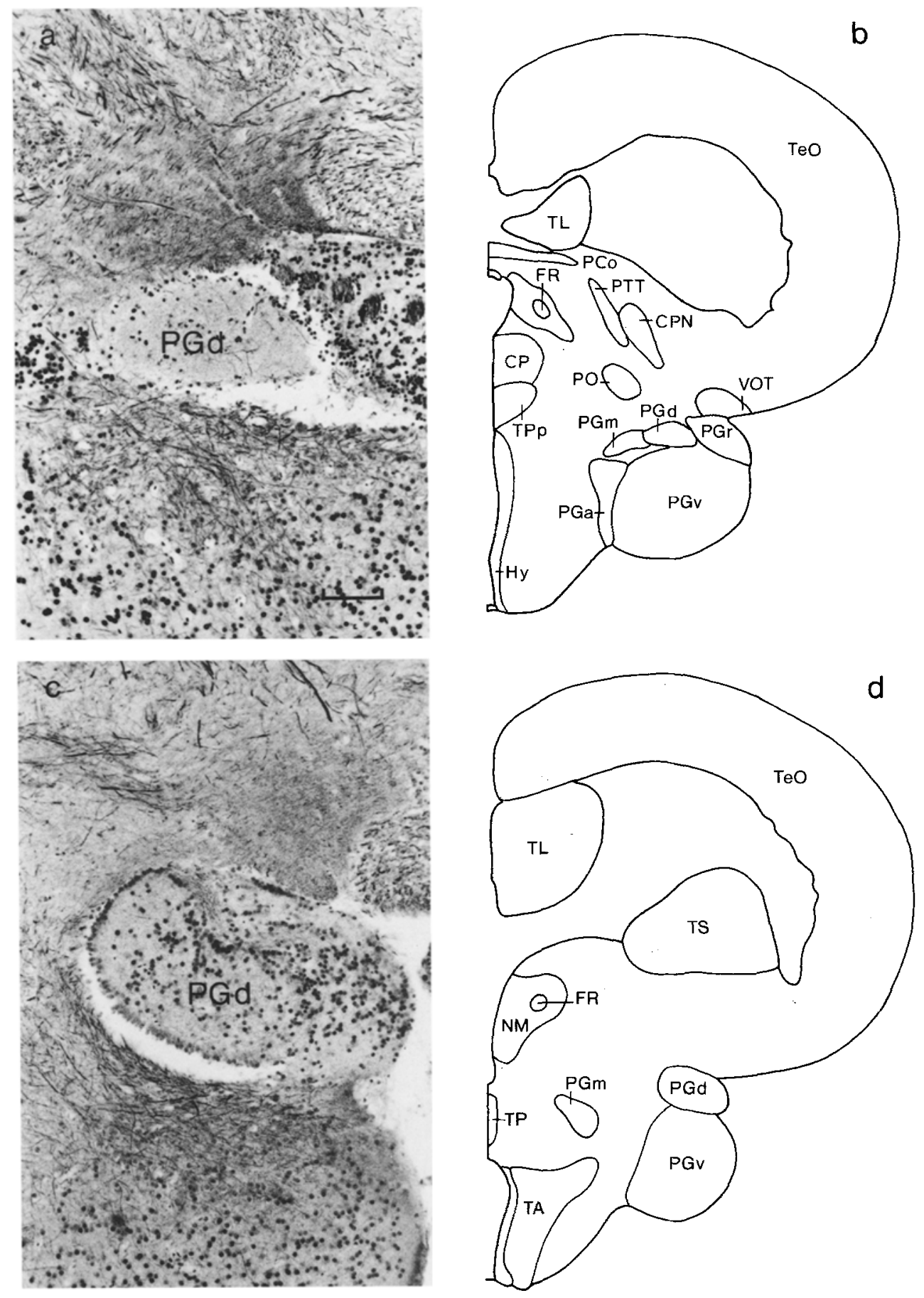

Fig. 2. Photomicrographs of silverstained cross-sections through the dorsal preglomerular nucleus in $P$. buchholzi $(\mathbf{a}, \mathbf{c})$ and line drawings of corresponding brain cross-sections (b,d). a, b Rostrally, the dorsal preglomerular nucleus is bordered laterally by the rostral preglomerular nucleus. c, d Caudally, the nucleus extends to the lateral surface of the brain. $C P$ Central posterior thalamic nucleus; $C P N$ central pretectal nucleus; $F R$ fasciculus retroflexus; $H y$ periventricular hypothalamus; $N M$ nucleus of the medial longitudinal fascicle; $P C o$ posterior commissure; $P G a$ anterior preglomerular nucleus; $P G d$ dorsal preglomerular nucleus; $P G m$ medial preglomerular nucleus; $P G r$ rostral preglomerular nucleus; $P G v$ ventral preglomerular nucleus; $P O$ posterior pretectal nucleus; $P T T$ preglomerulo-toral tract; $T A$ anterior tuberal nucleus; $\mathrm{TeO}$ tectum opticum; TL torus longitudinalis; TP posterior tuberal nucleus; $T P p$ periventricular nucleus of the posterior tuberculum; $T S$ torus semicircularis; $\mathrm{Va}$ valvula cerebelli; VOT ventrolateral optic tract: Bar: $0.05 \mathrm{~mm} ; \mathbf{a}, \mathbf{c}$ identical magnification

valvulae, locus coeruleus, reticular formation, inferior olive, nucleus of the descending trigeminal tract). Additionally, in P. buchholzi, many of the peripherally located neurons of the ipsilateral dorsal preglomerular nucleus were retrogradely labeled (Fig. 3a,b). No other preglomerular nuclei project to the cerebellum in $P$. buchholzi. In contrast to C. auratus, no cells were labeled in a position where a nucleus paracommissuralis would be expected.

\section{Discussion}

\section{Cerebellar connections in Carassius auratus}

The cerebellar connections in C. auratus have been documented in detail previously (Wullimann and Northcutt 1988, 1989). In these studies, both the minute paracommissural nucleus and the larger dorsal tegmental nucleus were demonstrated to project to the corpus cerebelli (Fig. 4a,b). However, none of the preglomerular nuclei in C. auratus were reported to project to the cerebellum. 

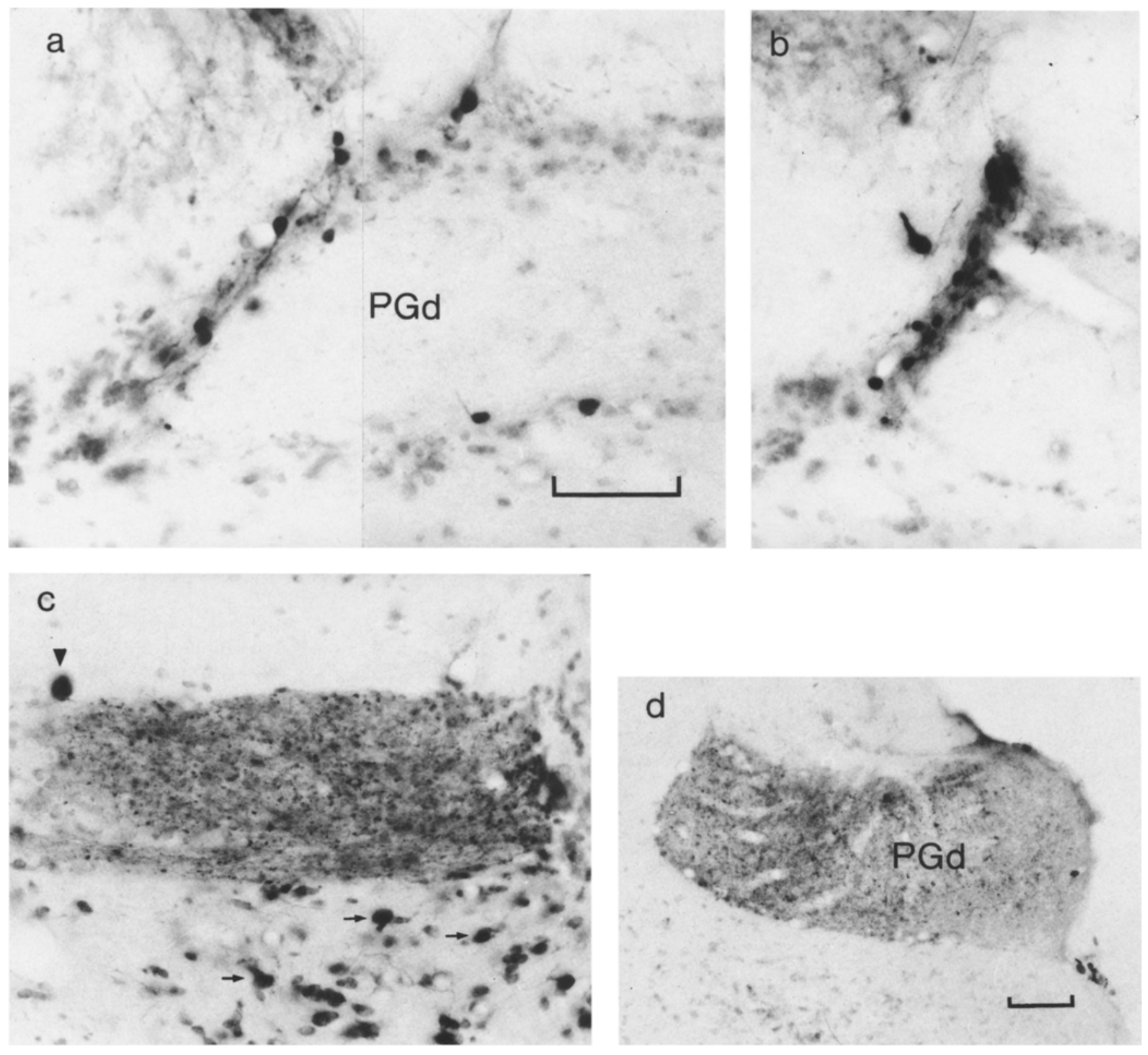

Fig. 3. Photomicrographs of cross-sections through the dorsal preglomerular nucleus of $P$. buchholzi after an HRP injection into the corpus cerebelli $(\mathbf{a}, \mathbf{b})$ or into the telencephalon $(\mathbf{c}, \mathbf{d})$. Rostral (a) and caudal (b) levels of the dorsal preglomerular nucleus with peripherally located neurons labeled retrogradely from the corpus cerebelli. Level of a corresponds to that shown in Fig. 2b. Rostral (c) and

caudal (d) levels of the dorsal preglomerular nucleus showing the terminal field after an HRP injection into the telencephalon. More ventrally, note the retrogradely labeled neurons (arrows point out three of them) in the ventral preglomerular nucleus in c. Arrowhead points to an artefact. $P G d$ Dorsal preglomerular nucleus. Bars: $0.05 \mathrm{~mm}$; a-c identical magnification

\section{Telencephalic connections in Carassius auratus}

Recently, telencephalic connections in the channel catfish Ictalurus punctatus have been reported (Striedter 1990). Since both $I$. punctatus and $C$. auratus belong to the ostariophysine teleosts, a detailed comparison appears to be appropriate. In general, telencephalic connections with the diencephalon and the mesencephalon in C.auratus are very similar to the ones in $I$. punctatus. Both species have extensive reciprocal telencephalic connections with the anterior preglomerular, the lateral preglomerular, and the posterior tuberal nuclei, as well as an afferent

connection to the telencephalon from the posterior thalamic nucleus.

Efferent projections exist in both species to the paracommissural, the ventrolateral thalamic, the anterior tuberal nuclei, the optic tectum, the region around the most caudal periventricular nucleus of the hypothalamus, the diffuse and central nuclei of the inferior lobe, and the nucleus of the torus lateralis. The tertiary gustatory nucleus (nucleus 'glomerulosus') in C. auratus as well as the homologous nucleus in $I$. punctatus lack telencephalic connections. More caudally located nuclei (dorsal tegmental nucleus, superior raphe, locus coeruleus) are not 

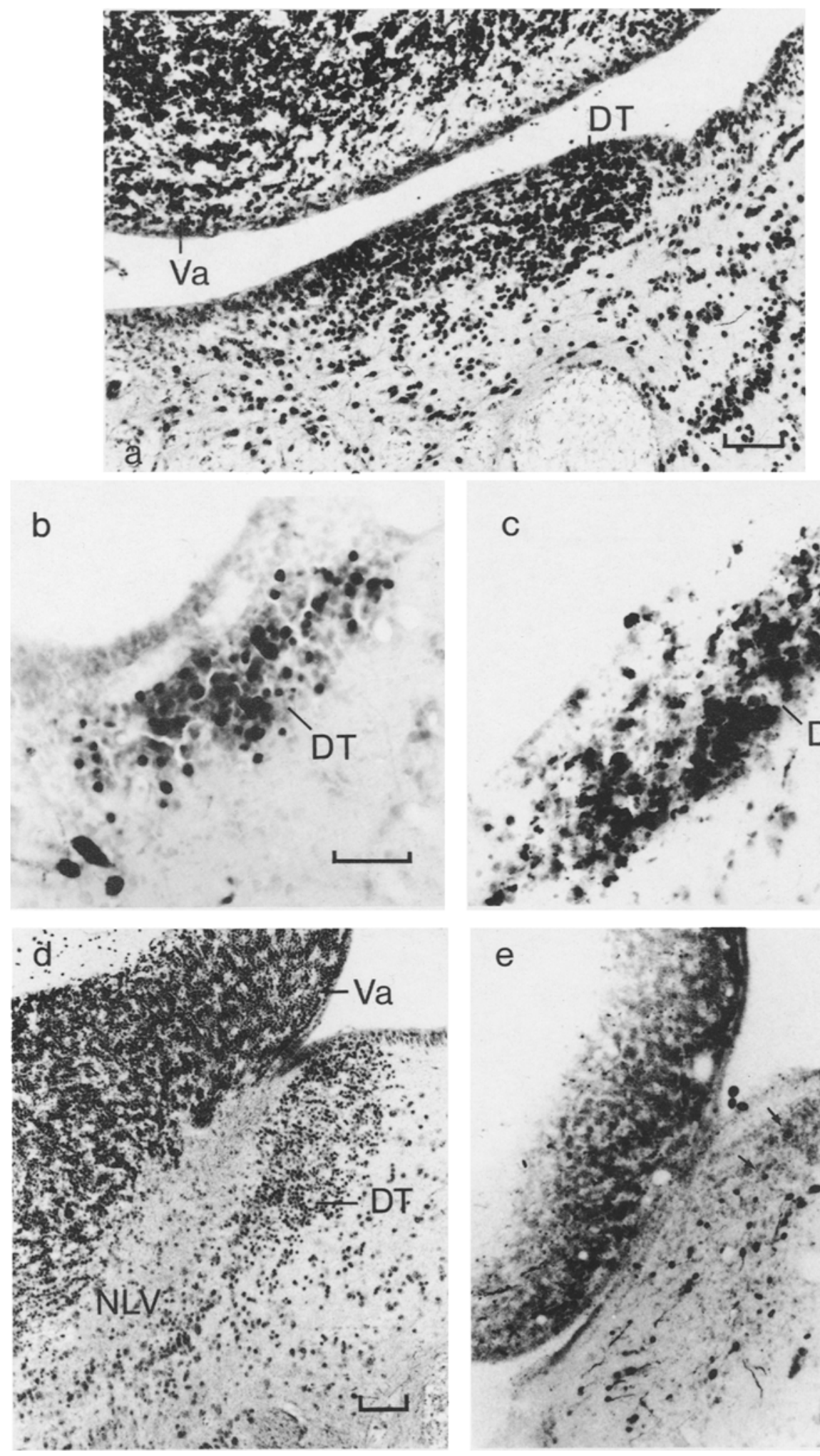
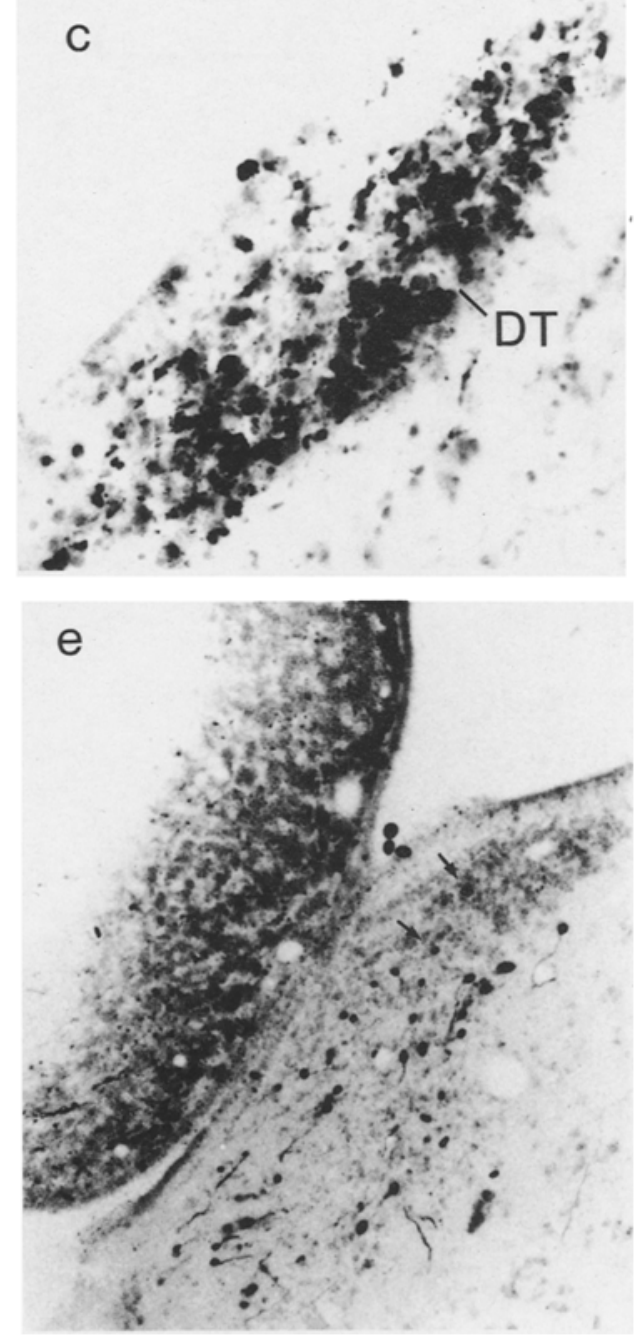

Fig. 4. Photomicrographs of crosssections through the dorsal tegmental nucleus of $C$. auratus $(\mathbf{a}-\mathbf{c})$ and $P$. buchholzi $(\mathbf{d}-\mathbf{e})$. a Histology of the nucleus in C. auratus in Bodian silver staining. Level of section is shown in Fig. 1e. b Retrogradely labeled neurons of the dorsal tegmental nucleus after HRP injection into the corpus cerebelli. c Large axo-somatic terminals on neurons in the dorsal tegmental nucleus after HRP injection into the telencephalon. d Histology of the dorsal tegmental nucleus in P. buchkolzi shown in a Bodian silver staining. Note the different histology of the nucleus lateralis valvulae. e Retrogradely labeled neurons of the dorsal tegmental nucleus (some are indicated by arrows) and of the nucleus lateralis valvulae after an HRP injection into the corpus cerebelli. Note also terminals within the granular layer of the valvula cerebelli. $D T$ Dorsal tegmental nucleus; $N L V$ nucleus lateralis valvulae; $V a$ valvula cerebelli. Bars: $0.1 \mathrm{~mm}$ in a; $0.05 \mathrm{~mm}$ in $b$ (c identical magnification), and $0.05 \mathrm{~mm}$ in $d$ ( $e$ identical magnification) covered by Striedter (1990), since his description of telencephalic connections in I. punctatus does not extend beyond mesencephalic levels.

There are also differences between $I$. punctatus and $C$. auratus. The latter species lacks ascending projections to the telencephalon originating in various anterior tuberal and inferior lobe nuclei. These nuclei also do not project to the telencephalon in the percomorph Sebastiscus marmoratus (Murakami et al. 1983). Thus, these telencephalic afferents likely represent uniquely derived specializations of the auditory and gustatory systems in I. punctatus (Striedter 1990). 


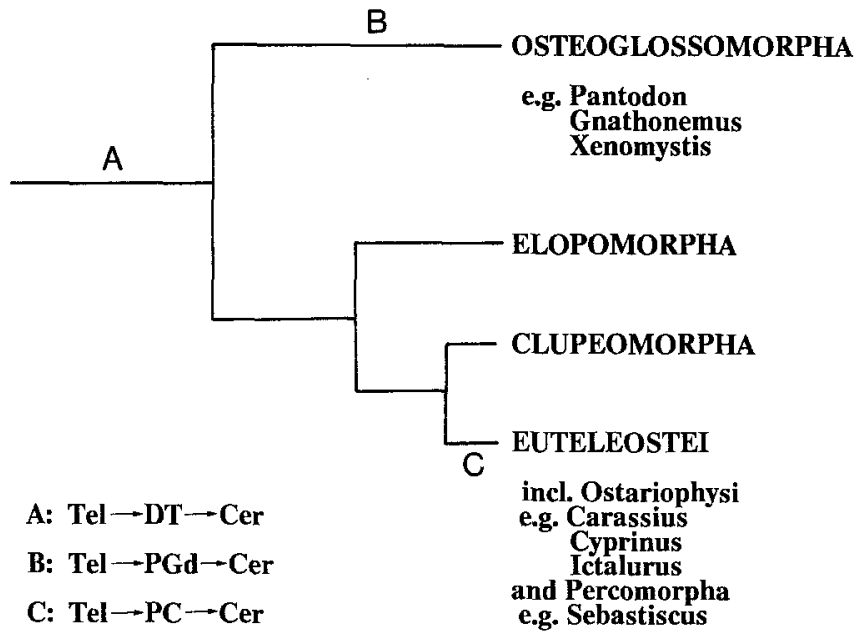

Fig. 5. Cladogram reveals phylogenetic relationships between the four extant teleost groups (after Lauder and Liem 1983). Teleost genera and higher taxa used in the text are indicated. $A, B$, and $C$ represent the three known pathways in teleosts from telencephalon $(\mathrm{Tel})$ to cerebellum $(\mathrm{Cer})$, which are relayed over either $(A)$ the dorsal tegmental nucleus $(D T),(B)$ the dorsal preglomerular nucleus $(P G d)$, or $(C)$ the paracommissural nucleus $(P C)$. The distribution of these pathways is plotted into the cladogram. Whereas $A$ appears to be plesiomorphic for teleosts, $B$ and $C$ are likely apomorphic for osteoglossomorphs and euteleosts, respectively

In contrast, the absence of ascending projections from dorsal and ventral thalamic nuclei in C. auratus, as compared to I. punctatus, may be due to the fact that not all telencephalic subdivisions were covered by the injections in $C$. auratus. This interpretation is further supported by the fact that telencephalic afferents from the dorsal thalamus have been reported before in C. auratus (Echteler and Saidel 1981). These differences, as well as an additional efferent projection to the habenula in I. punctatus, are not surprising, since the injections in $I$. punctatus comprised an entire telencephalic hemisphere. In this light, it is noteworthy that the telencephalic projection from the subglomerular nucleus seen in C. auratus is absent in I. punctatus (Striedter 1990).

In all teleost species which have been examined in detail, the preglomerular nuclei are heavily interconnected with the telencephalon (Murakami et al. 1983; Striedter 1990; Wullimann and Northcutt 1990). In C. auratus, the lateral preglomerular nucleus fades out at levels rostral to the one shown in Fig. 1a, whereas the anterior preglomerular nucleus, as originally described by Braford and Northcutt (1983), is enlarged and extends to the lateral surface of the brain. Still at this most rostral level, the anterior preglomerular nucleus is anterogradely and retrogradely labeled from the telencephalon in our preparations. This rostral portion of the anterior preglomerular nucleus may be homologous to our rostral preglomerular nucleus in $P$. buchholzi, which is obviously a nucleus separate from the anterior preglomerular nucleus in the latter species (Fig. 2a,b). A similar rostral preglomerular nucleus was described before in G. petersi (Wullimann and Northcutt 1990).
The ventral and dorsal preglomerular nuclei receive mechanosensory and electrosensory information from the torus semicircularis, respectively, and were first so designated in G. petersi by Wullimann and Northcutt (1990). Surprisingly, a dorsal preglomerular nucleus can also be recognized in $P$. buchholzi but its functional and phylogenetic origin is unclear (see below). Pending hodological confirmation of a mechanosensory toral input to the ventral preglomerular nucleus in P. buchholzi, the latter nucleus can be regarded to be homologous to the (mechanosensory) lateral preglomerular nucleus of other teleost species.

\section{Multiple indirect telencephalo-cerebellar pathways in teleosts}

The present findings on telencephalic and cerebellar connections in C. auratus and P. buchholzi confirm and also extend our view of the evolution of indirect telencephalocerebellar systems in teleosts:

(1) The pathway via the dorsal tegmental nucleus: Wullimann and Northcutt $(1988,1989)$ have identified two cell groups in the dorsal tegmental area which both project to the valvula and corpus cerebelli in $C$. auratus (Fig. 4b). The dorsal tegmental nucleus is composed of a tightly packed cell plate (Fig. 4a) located rostral and lateral to nucleus lateralis valvulae. The cells of the latter nucleus are more loosely arranged. The new data confirm the presence of two separate nuclei, since only the dorsal tegmental nucleus, but not the nucleus lateralis valvulae, receives a telencephalic input in C. auratus (Fig. 4c).

Two histologically distinct nuclei (dorsal tegmental nucleus, nucleus lateralis valvulae) can also be distinguished in P. buchholzi (Fig. 4d). The absence of a telencephalic projection to the dorsal tegmental nucleus in our HRP experiments is due to the fact that not all parts of the telencephalon were covered by the injections, since DiI injections into the lateral forebrain bundle of $P$. buchholzi resulted in the labeling of terminals in this nucleus. These terminals resemble closely those described previously in the dorsal tegmental nucleus of G. petersi (Wullimann and Northcutt 1990), i.e. they are very large axonal endings forming a basket around the neuronal somata.

As noted already, a telencephalo-recipient dorsal tegmental nucleus was also reported in G. petersi (Wullimann and Northcutt 1990). The neurons composing the former nucleus and the nucleus lateralis valvulae project to the corpus cerebelli in this osteoglossomorph species (Meek et al. 1986). In a second osteoglossomorph, Xenomystis nigri, a massive telencephalic projection also terminates in a dorsal tegmental nucleus, which is further said to project to the cerebellum (Nieuwenhuys and Meek 1990). Nieuwenhuys and Meek (1990) include this nucleus in the nucleus lateralis valvulae. HRP-injections into the nucleus lateralis valvulae of Cyprinus carpio which have recently been reported (Ito and Yoshimoto 1990), did not result in retrograde label in the telencephalon. In accordance with our results this suggests 
that the dorsal tegmental nucleus should not be included within the boundaries of nucleus lateralis valvulae.

Further, a dorsal tegmental nucleus projecting to the corpus cerebelli (Wullimann and Northcutt 1988) and receiving a telencephalic input was also reported for percomorphs (central gray of Vanegas and Ebbesson 1976; mesencephalic tegmentum of Murakami et al. 1983).

In summary, the presence of a telencephalo-cerebellar pathway via a dorsal tegmental nucleus in osteoglossomorphs, ostariophysines, and percomorphs suggests that this pathway is ancestral (plesiomorphic) for teleosts (Fig. 5).

(2) The pathway via the dorsal preglomerular nucleus: The dorsal preglomerular nucleus of G. petersi has been interpreted as an additional subdivision of the preglomerular area which is not present in other teleosts (Wullimann and Northcutt 1987, 1990) for two main reasons: (i) It receives ascending lateral line information (i.e., electrosensation) from the torus semicircularis, as do other preglomerular nuclei. (ii) It receives efferents from the telencephalon, as do other preglomerular nuclei. However, a separate dorsal preglomerular nucleus receiving efferents from, but not projecting to, the telencephalon, and additionally projecting to the cerebellum, has not been described in teleosts other than $G$. petersi. Therefore, Wullimann and Northcutt $(1987,1990)$ interpreted the dorsal preglomerular nucleus and its telencephalocerebellar circuit as a derived feature which evolved in correlation with the electrosensory modality in mormyrids.

Moreover, in the present study, we found the non-electrosensory osteoglossomorph $P$. buchholzi to possess a dorsal preglomerular nucleus which receives a telencephalic input and projects to the cerebellum. As neither ostariophysine nor percomorph teleosts have a comparable preglomerular telencephalo-cerebellar relay center, the latter is probably autapomorphic for some or all osteoglossomorphs (Fig. 5). If, alternatively, this telencephalo-cerebellar pathway were plesiomorphic for teleosts, it must have been lost in more derived teleosts, such as euteleosts, where the circuit is replaced by a homoplasous pathway via the nucleus paracommissuralis.

(3) The pathway via nucleus paracommissuralis: This telencephalo-cerebellar pathway via the nucleus paracommissuralis was initially described by Karten and Finger (1976) in I. punctatus and later confirmed in several additional euteleosts by Ito et al. (1982). Wullimann and Northcutt (1988) surveyed the presence of nucleus paracommissuralis in various teleosts based on normal histology and concluded that this nucleus only evolved in euteleosts (Fig. 5). The present data support this interpretation. In the osteoglossomorph $P$. buchholzi, a nucleus paracommissuralis can neither be discerned histologically, nor on the basis of telencephalic or cerebellar connections.

In $C$. auratus, Wullimann and Northcutt (1988) tentatively termed a small cell group dorsally to the posterior commissure "nucleus paracommissuralis", since it projects to the corpus cerebelli. As expected, this nucleus also receives an input from the telencephalon confirming its identification.

The results obtained in P. buchholzi and in C. auratus corroborate the interpretation that a nucleus paracommissuralis and the related telencephalo-cerebellar pathway are derived features (apomorphic) for euteleosts (Fig. 5).

\section{Conclusion}

The present findings indicate that three telencephalocerebellar pathways have developed at different stages during the evolution of teleosts (Fig. 5). The dorsal preglomerular nucleus and the nucleus paracommissuralis, acting as a relay between telencephalon and cerebellum, probably evolved anew in osteoglossomorphs and in euteleosts, respectively. A dorsal tegmental nucleus acting as such a relay appears to be plesiomorphic for teleosts. Unfortunately, cerebellar and telencephalic connections are unknown in nonteleost actinopterygians. Telencephalic efferents have not been described in sufficient detail at the nuclear level in cartilaginous fishes (Ebbesson 1980). However, many tegmental nuclei have been documented to project to the cerebellum in one cartilaginous fish (Fiebig 1988). It is thus not unlikely that a homologon of the teleostean dorsal tegmental nucleus exists already in chondrichthyans.

The multiple telencephalo-cerebellar pathways of teleosts represent a striking convergence to the telencephalo-pontine systems in birds and mammals. Similar to these vertebrates, the cerebellum is massively enlarged and differentiated in most teleosts. The indirect telencephalic input may be one reason for this. Given the fact that teleosts show more than half of the species diversity in extant vertebrates, it is not surprising that they - similar to birds and mammals - evolved telencephalo-cerebellar pathways independently several times during evolution. Since the present paper establishes the systematic distribution and the neuroanatomical and hodological configuration of these systems in teleosts, a functional investigation is now within reach. It will eventually reveal to what degree these pathways convey a similar telencephalic influence on the cerebellum in teleosts as compared to birds and mammals.

Acknowledgements. We thank Simona Hellbach and Kristina Wullimann for technical assistance. Further, M.F.W. would like to thank Ulrike Niemann for some photographic aid. This study was supported by the Deutsche Forschungsgemeinschaft.

\section{References}

Braford MR Jr, Northcutt RG (1983) Organization of the diencephalon and pretectum of the ray-finned fishes. In: Davis $R$, Northcutt RG (eds) Fish neurobiology, vol 2. University of Michigan Press, Ann Arbor, pp 117-163

Bullock TH, Bodznick DA, Northcutt RG (1983) The phylogenetic distribution of electroreception: evidence for convergent evolution of a primitive vertebrate sensory modality. Brain Res 6:2546 
Ebbesson SOE (1980) On the organization of the telencephalon inelasmobranchs. In: Ebbesson SOE (ed) Comparative neurology of the Telencephalon. Plenum Press, New York, pp 1-16

Ebbesson SOE, Hansel M, Scheich H (1981) An 'on the slide' modification of the DeOlmos-Heimer-HRP method. Neurosci Lett $22: 1-14$

Echteler SM, Saidel WM (1981) Forebrain connections in the goldfish support telencephalic homologies with land vertebrates. Science 212:683-685

Fiebig E (1988) Connections of the corpus cerebelli in the thornback guitarfish, Platyrhinoidis triseriata (Elasmobranchii): a study with WGA-HRP and extracellular granule cell recording. J Comp Neurol 268:567-583

Finger TE, Bell CC, Russell CJ (1981) Electrosensory pathways to the valvula cerebelli in mormyrid fish. Exp Brain Res 42:23-33

Hanker JS, Yates PE, Metz CB, Rustioni A (1977) A new specific, sensitive and non-carcinogenic reagent for the demonstration of horseradish peroxidase. Histochem J 9:789-792

Ito $\mathrm{H}$, Yoshimoto $\mathrm{M}$ (1990) Cytoarchitecture and fiber connections of the nucleus lateralis valvulae in the carp (Cyprinus carpio). J Comp Neurol 298:385-399

Ito H, Murakami T, Morita Y (1982) An indirect telencephalo-cerebellar pathway and its relay nucleus in teleosts. Brain Res 249:1-13

Karten HJ, Finger TE (1976) A direct thalamo-cerebellar pathway in pigeon and catfish. Brain Res 102:335-338

Lauder GV, Liem KF (1983) The evolution and interrelationships of the actinopterygian fishes. Bull Mus Comp Zool 150:95-197

Meek J, Nieuwenhuys R, Elsevier D (1986) Afferent and efferent connections of cerebellar lobe $\mathrm{C} 3$ of the mormyrid fish Gnathonemus petersi: an HRP study. J Comp Neurol 245:342358
Murakami T, Morita Y, Ito H (1983) Extrinsic and intrinsic fiber connections of the telencephalon in a teleost, Sebastiscus marmoratus. J Comp Neurol 216:115-131

Nieuwenhuys R, Meek J (1990) The telencephalon of actinopterygian fishes. In: Jones EG, Peters A (eds) Cerebral cortex, vol 8a: Comparative structure and evolution of cerebral cortex, part 1. Plenum Press, New York London, pp 31-73

Roth G, Wullimann MF (1993) Die Evolution der Nervensysteme und der Sinnesorgane. In: Dudel J, Menzel R, Schmidt RF (eds) Lehrbuch der Neurowissenschaft. VCH, Weinheim (in press)

Striedter GF (1990) The diencephalon of the channel catfish, Ictalurus punctatus. II. Retinal, tectal, cerebellar and telencephalic connections. Brain Behav Evol 36:355-377

Vanegas H, Ebbesson SOE (1976) Telencephalic projections in two teleost species. J Comp Neurol 165:181-196

Wullimann MF, Meyer DL (1990) Phylogeny of putative cholinergic visual pathways through the pretectum to the hypothalamus in teleost fish. Brain Behav Evol 36:14-29

Wullimann MF, Northcutt RG (1987) Telencephalic and retinal projections allow reinterpretation of the diencephalon in mormyrids. Soc Neurosci Abstr 13:130

Wullimann MF, Northcutt RG (1988) Connections of the corpus cerebelli in the green sunfish and the common goldfish: A comparison of perciform and cypriniform teleosts. Brain Behav Evol $32: 293-316$

Wullimann MF, Northcutt RG (1989) Afferent connections of the valvula cerebelli in two teleosts, the common goldfish and the green sunfish. J Comp Neurol 289:554-567

Wullimann MF, Northcutt RG (1990) Visual and electrosensory circuits of the diencephalon in mormyrids: an evolutionary perspective. J Comp Neurol. 297:537-552

Wullimann MF, Roth G (1992) Similar telencephalo-cerebellar circuitry in electrosensory and other osteoglossomorph teleost fishes. Eur J Neurosci 5 [Suppl]:290 